\title{
Auroral kilometric radiation from a nonstationary thin plasma cavity
}

\author{
T. M. Burinskaya ${ }^{1}$ and J.-L. Rauch ${ }^{2}$ \\ ${ }^{1}$ Space Research Institute of RAS, Profsoyuznaya str. 84/32, Moscow, 117997, Russia \\ ${ }^{2}$ Laboratoire de Physique et Chimie de L'Environnement et de L'Espace, UMR6115 CNRS - Université d'Orléans, \\ 3A Avenue de la Recherche Scientifique, France
}

Correspondence to: T. M. Burinskaya (tburinsk@iki.rssi.ru)

Received: 3 February 2012 - Revised: 11 June 2012 - Accepted: 12 June 2012 - Published: 25 July 2012

\begin{abstract}
Results obtained using a waveguide model of the AKR generation in thin plasma cavities are presented. Taking into account the occurrence of low frequency plasma motion in the regions of the AKR generation, we have considered a wave escape from a thin plasma cavity with adiabatically slowly varying width, and show that there can exist localized regions of instability from which the extraordinary Xmode waves, growing in time, can be radiated outwards. It has been found that waves, propagating quasi-tangentially to the source frontiers, have the maximum growth rate and escape outward most efficiently, which is in accordance with experimental observations.
\end{abstract}

Keywords. Electromagnetics (Wave propagation) - Magnetospheric physics (Auroral phenomena; Plasma waves and instabilities)

\section{Introduction}

The electron cyclotron maser instability (Wu and Lee, 1979) is widely accepted to be the fundamental mechanism responsible for generation of the terrestrial auroral kilometric radiation (AKR) and similar radio emissions from the solar system planets having their own magnetic field: Jupiter, Saturn, Uranus, and Neptune (Zarka, 1998; Farrell et al., 2004).

The AKR is strongly polarized emission mainly propagating as the extraordinary X-mode waves (Panchenko et al., 2008). According to the Viking spacecraft observations, AKR sources are thin cavities oriented tangentially to the auroral oval and aligned with the background magnetic field (Louarn and Le Quéau, 1996a; Ergun et al., 1998). They have a small latitudinal width $(10 \mathrm{~km}-100 \mathrm{~km})$ as compared with their longitudinal extent $(\sim 2000 \mathrm{~km})$. Plasma of AKR sources is tenuous with electron population essentially composed of energetic particles with a horseshoe velocity distribution (Ergun et al., 2000). The free energy that drives the cyclotron maser instability is associated with a positive slope $\partial f / \partial p_{\perp}$ in the horseshoe distribution. A peculiar feature of the electron distributions observed inside the sources is an accumulation of particles with high transverse energies of the order of several $\mathrm{KeV}$ but low parallel velocities. AKR sources are separated from the denser and colder surrounding plasma by sharp density gradients with typical length scale of a few hundred meters.

Taking into account the above-mentioned experimental results, Louarn and Le Quéau (1996b) were the first who suggested the plane waveguide model of the AKR generation in thin plasma cavities (Fig. 1). Using this model, a general dispersion relation was obtained for wave generation in an arbitrary direction relative to the magnetic field (Burinskaya and Rauch, 2007).

It was found that there is a preferential direction of wave excitation in the plane transverse to the background magnetic field, namely, in the vicinity of the y-axis tangential to the source frontiers. This fact is completely consistent with experimental data. Observations made onboard the Viking spacecraft have revealed that the AKR radiating diagram is anisotropic in the plane perpendicular to the geomagnetic field, and radiation is preferentially emitted quasitangentially to the source frontiers (Louarn and Le Quéau, 1996a). The recent analysis of Cluster observations of the AKR emissions has shown that the AKR angular beaming pattern is narrowly confined to a plane containing the magnetic field vector at the source and tangent to a circle of 


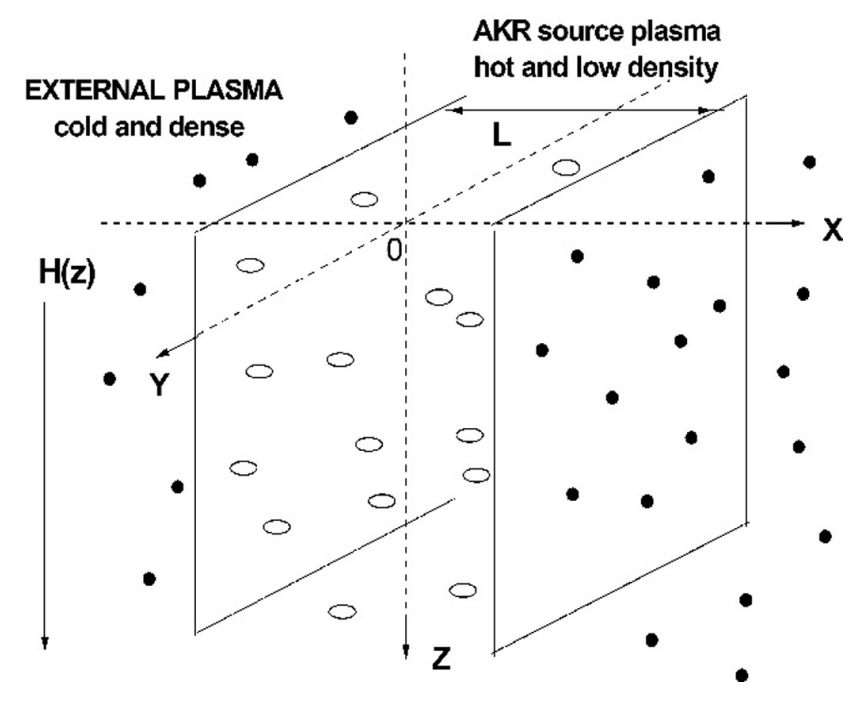

Fig. 1. Geometry of the AKR source model.

constant latitude (Mutel et al., 2008). Therefore, it seems that the waveguide model is a good tool for studying of AKR emissions generated inside thin cavities.

However, there is a long-standing problem in studies of cyclotron maser instability in a localized space region with a reduced density: how the radiation generated below the cutoff frequency of the external cold plasma would escape from a source or, in other words, gets onto the branch of the cold plasma dispersion. The most plausible hypothesis is that plasma and magnetic field inhomogeneities, as well as time dependent processes, are of primary importance in the understanding of the AKR escape from a source region. The role of magnetic field gradients was qualitatively discussed by Louarn and Le Quéau (1996b), and some interesting quantitative estimates were made by Cairns et al. (2008), but a comprehensive analysis of this problem has not been performed yet. Taking into account that low frequency waves commonly exist in the AKR generation region (Lund, 2010), we develop a nonstationary waveguide model and show that incorporation of time-dependent processes into the waveguide model enables the AKR emissions generated inside a cavity to escape from it. In this paper, a thin plasma cavity with adiabatically slowly varying width is considered. Our objective is to find solutions in which the extraordinary $\mathrm{X}$-mode waves growing inside a source can be directly connected to the external X-mode waves attributed to the cold plasma dispersion.

\section{Model of the waveguide with slowly varying width}

We use a model shown in Fig. 1 in which the AKR source is considered to be a plane waveguide that has the width $L$ along the $\mathrm{x}$-axis and is infinite in the $\mathrm{y}$ - and $\mathrm{z}$-directions. The $\mathrm{z}$-axis is directed along the background magnetic field. Un- der the assumption that plasma parameters vary on a characteristic spatial scale much greater than a source width, the plasma inside and outside a source can be treated as homogeneous. Because of the complexity of the dispersion relation for a horseshoe distribution, a ring distribution of the form $f\left(v_{\mathrm{z}}, v_{\perp}\right)=\left(2 \pi v_{\perp 0}\right)^{-1} \delta\left(v_{\perp}-v_{\perp 0}\right) \delta\left(v_{z}-v_{0}\right)$ is taken inside a source. Here, $v_{0}$ is the directed electron velocity along the magnetic field. Although such a distribution function is idealized, it takes into account relativistic effects and does not significantly modify the results obtained with the use of more realistic distributions, as was shown in Le Quéau and Louarn (1989). It is suggested that the plasma outside a source is cold. The width of transition layer between the low density hot plasma inside the source and the external denser cold plasma is assumed to be much less than the wavelength of the excited waves. The ions are presumed to be immobile and to play the role of a neutralizing background. Since relativistic effects play a key role in cyclotron maser instabilities, the relativistic dielectric tensor elements are used. For the investigation of the wave generation and its propagation in an arbitrary direction inside a source with a finite extension, it is necessary to consider the general asymmetric structure of electromagnetic fields inside a source, as shown in Burinskaya and Rauch (2007) where the relevant dispersion relation is found from the continuity conditions for electromagnetic field components $H_{\mathrm{z}}, E_{\mathrm{z}}, H_{\mathrm{y}}, E_{\mathrm{y}}$ at the waveguide frontiers. As may be expected, solutions to the dispersion equation constitute a discrete family of waveguide eigenmodes, each of which can be assigned an integer $n=1+k_{\mathrm{x}} L / \pi$, where $L$ is a source width and $k_{\mathrm{x}}$ is a wave vector component in the $\mathrm{x}$ direction. For each $n$ value, the real and imaginary parts of wave frequency are found by numerically solving the dispersion relation as functions of wave vector components $k_{\mathrm{y}}$ and $k_{z}$. The results obtained in Louarn and Le Quéau (1996b), Burinskaya and Rauch (2007) have shown that the real parts of the refractive index calculated for the eigenmodes with a positive value of the growth rate are less than unit inside a source region, and more than unit outside a source. Using the terminology for the cold plasma, it is possible to say that the internal part of these solutions is similar to the X-mode waves, and the external part is similar to the Z-mode waves. It worth to note that there is an additional branch of the dispersion relation in a homogeneous relativistic plasma with hot electrons characterized by the same parameters as the AKR source plasma (Louarn and Le Quéau, 1996b; Cairns et al., 2008). This branch with a negative group velocity takes place in a limited range of wave numbers, and connects together, two branches similar to the $\mathrm{X}$-mode and Z-mode waves. In the cold background plasma outside of a source region two branches corresponding to the X-mode and Z-mode waves are far apart on a dispersion relationship diagram.

In the present paper, we consider a waveguide with adiabatically slowly varying width, and numerically solve the dispersion relation on the condition that the number of particles inside a waveguide, as well as the magnetic flux and 


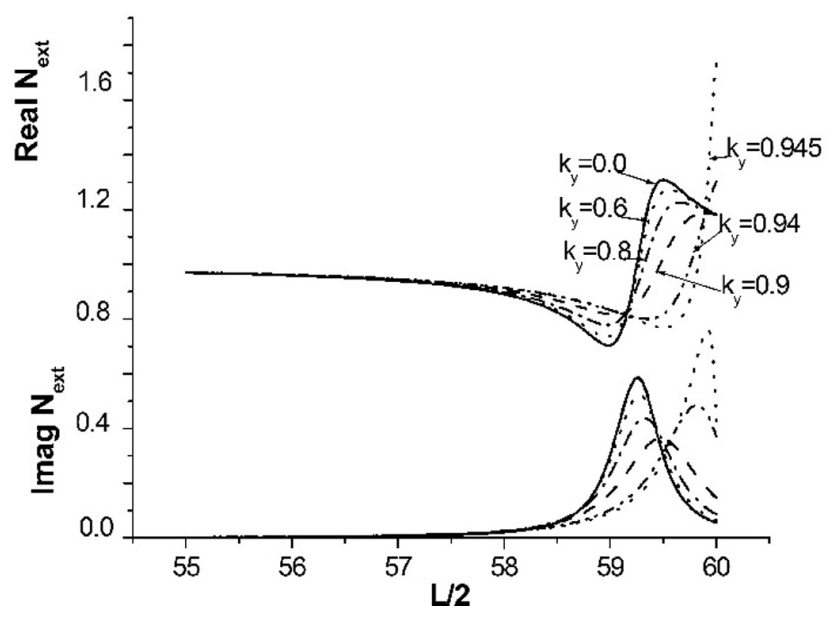

Fig. 2. Real and imaginary parts of the external index as functions of the dimensionless half-width of the source for the eigenmode $n=12$ with different values of $k_{y}$ normalized to $\omega_{0} / c$ and marked on the top part of this figure. The type of lines corresponding to defined values of $k_{\mathrm{y}}$ is the same for top and bottom curves.

the first adiabatic invariant, is constant. In what follows, we present results for the range of parameters adapted to the AKR generation region at altitudes where the electron gyrofrequency $f_{\mathrm{c}}=200 \mathrm{kHz}$. The electron energy and density inside the source region are taken $\sim 4 \mathrm{KeV}$ and $1 \mathrm{~cm}^{-3}$, respectively, and the background plasma density outside the source is $\sim 5 \mathrm{~cm}^{-3}$. The solutions of the dispersion equation are shown in Fig. 2 as the waveguide half-width dependence of the real and imaginary parts of the external refractive index $N_{\text {ext }}=k_{\text {out }} c / \omega$, where $c$ is the speed of light, $k_{\text {out }}$ and $\omega$ are a wavenumber and a frequency of the waves outgoing from a source. Calculations are made for the eigenmodes $n=12$ with $k_{\mathrm{z}}=0$ and different values of $k_{\mathrm{y}}$ normalized to $\omega_{0} / c$ ( $\omega_{0}$ is the electron cyclotron frequency in the background plasma). The values of $k_{\mathrm{y}}$ are marked on the top part of this figure, and the type of lines corresponding to defined values of $k_{\mathrm{y}}$ is the same for top and bottom curves. The source halfwidth is normalized to $c / \omega_{0}$, and is varied between 60 and 55 in dimensionless units corresponding to $15 \mathrm{~km}$ and $13.75 \mathrm{~km}$ in dimensional values for the plasma parameters mentioned above. Calculations were performed beginning with the dimensionless half-width equal to 60 when the real part of the external index is greater than unity for all solutions. In other words, there is a leakage only of Z-mode waves from the source. Since the source width is reduced, the real part of the external index rapidly decreases for the waves propagating nearly tangentially to waveguide frontiers, $k_{\mathrm{y}}>0.9$, and a contraction smaller than one percent gives the chance to leak for these waves outside of the X-mode waves, $N_{\mathrm{ext}}<1$. In Fig. 3 the real part of the external index is shown against the angle of wave propagation $\theta$ between the $y$-axis and a wave vector for the moment of time when a dimensionless source half-width was narrowed to $L / 2=59.4$. When a $2 \%$

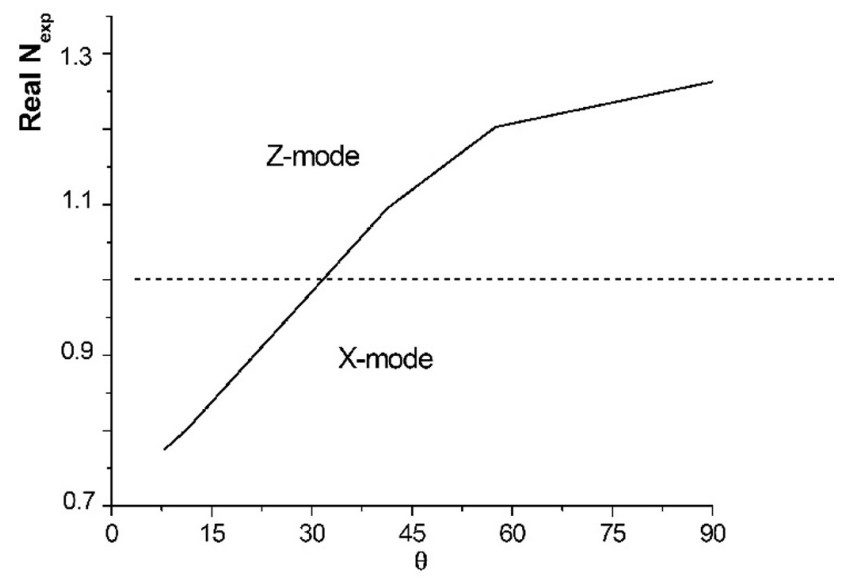

Fig. 3. Real part of the external index as a function of the angle of wave propagation $\theta$ between the $y$-axis and a wave vector of the outgoing waves for the moment of time when a dimensionless source half-width is equal to 59.4 .

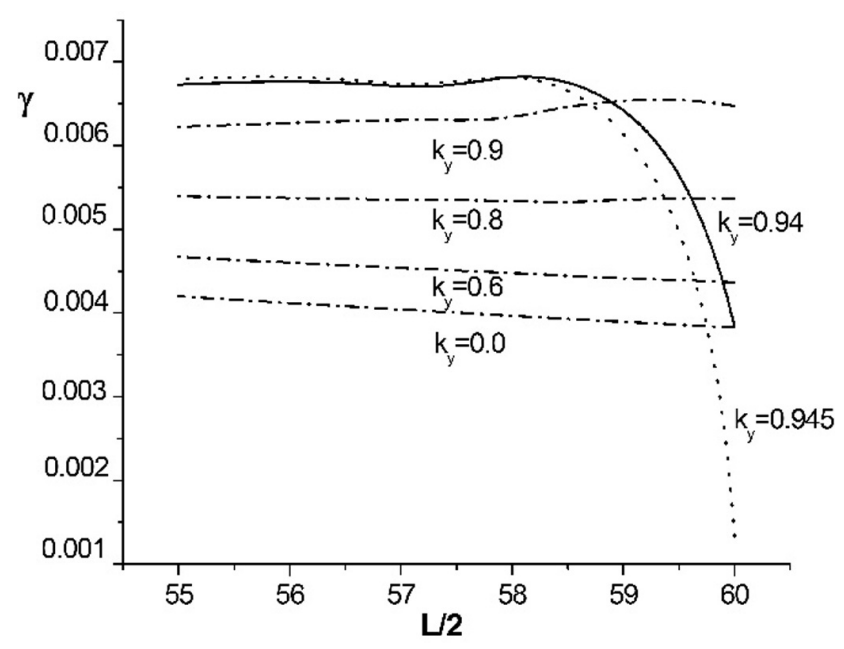

Fig. 4. Growth rate normalized to $\omega_{0} / c$ as a function of the dimensionless half-width for the eigenmode $n=12$ with different values of $k_{\mathrm{y}}$ normalized to $\omega_{0} / c$ and marked on the plot.

reduction in width is achieved, the real part of the external index becomes smaller than unity for all eigenmodes under consideration. With a further width narrowing, the imaginary part of the external index vanishes and its real part smaller than unity tends to unity, and it is possible to say that there is a leakage of the X-mode waves on the branch of the cold plasma dispersion.

The results obtained in the frame of the stationary waveguide model have shown that there is a limiting value of $k_{\mathrm{y}}$ found from $c \sqrt{k_{\mathrm{x}}^{2}+k_{\mathrm{y}}^{2}+k_{\mathrm{z}}^{2}}=\omega_{\mathrm{c}}$ for the growing eigenmodes $\left(\omega_{\mathrm{c}}\right.$ is the electron cyclotron frequency inside a source). The growth rate of cyclotron maser instability rises with increasing $k_{\mathrm{y}}$ if $k_{\mathrm{y}}$ is smaller than its limiting value, and rapidly reduces to zero if $k_{\mathrm{y}}$ is close to it (Burinskaya 


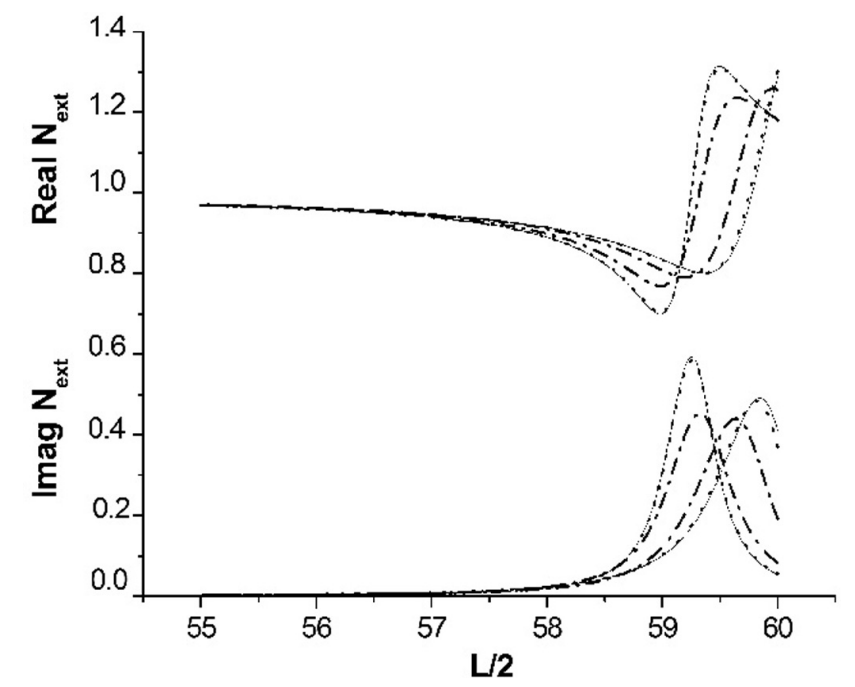

Fig. 5. Real and imagine parts of the external refractive index versus the dimensionless source half-width for eigenmodes with different $n$. Light solid lines correspond to the eigenmodes $n=4$ with different $k_{\mathrm{y}}$ : left $k_{\mathrm{y}}=0$; right $k_{\mathrm{y}}=0.98$. Dotted lines correspond to $n=12$ : left $k_{\mathrm{y}}=0$; right $k_{\mathrm{y}}=0.94$. Dash-dotted lines correspond to $n=33$ : left $k_{\mathrm{y}}=0$; right $k_{\mathrm{y}}=0.5$.

and Rauch, 2007). The limiting value of $k_{\mathrm{y}}$ is proportional to $\omega_{\mathrm{c}} / c$. Therefore, the small increase of the magnetic field inside a source due to its width contraction can lead to pronounced changes in the growth rate of waves with $k_{\mathrm{y}}$ close to the limiting value. The growth rate normalized to $\omega_{0} / c$ as a function of the dimensionless half-width is shown in Fig. 4 for the eigenmode $n=12$ with different values of $k_{\mathrm{y}}$ normalized to $\omega_{0} / c$. It is clearly seen that, during the waveguide contraction, the growth rate of the waves propagating very close to the y-axis $\left(k_{\mathrm{y}}>0.9\right)$ rises steeply at the initial stage and remains the greatest upon further width compression.

The results shown in Figs. 2-4 are obtained for the eigenmode $n=12$. For comparison, in Fig. 5 the real and imagine parts of the external refractive index are shown versus the dimensionless source half-width for three eigenmodes with different $n$. Light solid lines correspond to $n=4$ with different $k_{\mathrm{y}}$ : left $k_{\mathrm{y}}=0$; right $k_{\mathrm{y}}=0.98$. Dotted lines correspond to $n=12$ : left line $k_{\mathrm{y}}=0$; right line $k_{\mathrm{y}}=0.94$; and dashdotted lines to $n=33$ : left $k_{\mathrm{y}}=0$; right $k_{\mathrm{y}}=0.5$. It is apparent from this figure that the results obtained for the eigenmodes $n=4$ and $n=12$ are very close to each other. From our calculations, it follows that eigenmodes with $n<15$ and $k_{\mathrm{y}}>0.9$ are the first that can escape from the source as the $\mathrm{X}$-mode waves, and the width contraction on the order of $5 \%$ provides a leakage of all waves generated inside the source essentially on the branch of the cold plasma dispersion.

\section{Discussion}

We have considered a wave escape from a thin plasma cavity with adiabatically slowly varying width. It has been shown that it is possible to have a localized unstable region inside a cavity, from which outward propagating X-mode waves are generated because of the increasing of the magnetic field and plasma density inside the source region. For the parameters used above, it is enough to increase the magnetic field inside a cavity due to its contraction by some percent and, the main part of waves generated inside a source would leak out on the $\mathrm{X}$-mode branch of the cold plasma dispersion. It has been found that waves propagating quasi-tangentially to the source frontiers have the maximum growth rate and most efficiently escape outward, which is in accordance with experimental observations. Waves inside the source region grow in amplitude due to the development of the electron cyclotron maser instability, and as a consequence, amplitudes of the outgoing waves are also increased. The bandwidth of emitting waves depends on the degree of width compression, and its value is determined as the range of frequencies generated between two moments of time: the first must be taken when a leakage of the X-mode waves becomes possible, and the second is a final point of width contraction depending on amplitudes of low frequency waves in the region of AKR generation. It has been found that, during a width contraction, the intensity of the escaping radiation increases due to the increasing growth rate of cyclotron maser instability. During a width expansion, the intensity of the outgoing waves decreases. It is interesting to note that a similar correlation between the AKR power and low frequency oscillations of the magnetic field has been observed (Uozumi et al., 2011). For more detailed comparison with experimental data, it is also necessary to take into account the magnetic field and plasma inhomogeneities in the region of the AKR generation. Mutel et al. (2008) estimated the wave refraction outside the source by the denser magnetospheric plasma using the cold plasma dispersion. However, for the calculations of wave generation and propagation inside a source region, as well as their exit from a cavity, it is necessary to use a dispersion relation for the relativistic plasma with hot electrons, because a wave propagation in the presence of a distribution unstable to a cyclotron maser instability may be significantly different from that in a stable plasma (Cairns et al., 2008). The problems of the AKR generation and propagation in an inhomogeneous plasma in the presence of low frequency oscillations are under our current investigation. The main point to be taken from the present paper is that the existence of low frequency waves may play a key role in the AKR escape from the region of its generation.

Acknowledgements. This work was supported by RFBR grant 1002-93115 and 12-02-92614, and grant of Leading Scientific Schools of Russian Federation HIII623.2012.2.

Topical Editor R. Nakamura thanks three anonymous referees for their help in evaluating this paper. 


\section{References}

Burinskaya, T. M. and Rauch, J. L.: Waveguide regime of cyclotron maser instability in plasma regions of depressed density, Plasma Phys. Rep., 33, 28-37, doi:10.1134/S1063780X07010047, 2007.

Cairns, R. A., Vorgul, I., and Bingha, R.: Cyclotron maser radiation from an inhomogeneous plasma, Phys. Rev. Lett., 101, 215003, doi:10.1103/PhysRevLett.101.215003, 2008.

Ergun, R. E., Carlson, C. W., McFadden, J. P., Mozer, F. S., Delory, G. T., Peria, W., Chaston, C. C., Temerin, M., Elphic, R., Strangeway, R., Pfaff, R., Cattell, C. A., Klumpar, D., Shelley, E., Peterson, W., Moebius, E., and Kistler, L.: FAST satellite observations of electric field structures in the auroral zone, Geophys. Res. Lett., 25, 2025-2028, 1998.

Ergun, R. E., Carlson, C. W., McFadden, J. P., Delory, G. T., Strangeway, R. J., and Pritchett, P. L.: Electron-cyclotron maser driven by charged-particle acceleration from magnetic fieldaligned electric fields, Astrophys. J., 538, 456-466, 2000.

Farrell, W. M., Kaiser, M. L., Kurth, W. S., Desch, M. D., Gurnett, D. A., Hospadarsky, G. B., and MacDowall, R. J.: Remote sensing of possible plasma density bubbles in the inner Jovian dayside magnetosphere, J. Geophys. Res., 109, A09S14, doi:10.1029/2003JA010130, 2004.

Le Quéau, D. and Louarn, P.: Analytical study of the relativistic dispersion: application to the generation of the auroral kilometric radiation, J. Geophys. Res., 94, 2605-2616, 1989.
Louarn, P. and Le Quéau, D.: Generation of the auroral kilometric radiation in plasma cavities-I. Experimental study, Planet. Space Sci., 44, 199-210, 1996a.

Louarn, P. and Le Quéau, D.: Generation of the auroral kilometric radiation in plasma cavities-II. The cyclotron maser instability in small size sources, Planet. Space Sci., 44, 211-224, 1996b.

Lund, E. J.: On the dissipation scale of broadband ELF waves in the auroral region, J. Geophys. Res., 115, A01201, doi:10.1029/2009JA014545, 2010.

Mutel, R. L., Christopher, I. W., and Pickett, J. S.: Cluster multispacecraft determination of AKR angular beaming, Geophys. Res. Lett., 35, L07104, doi:10.1029/2008GL033377, 2008.

Panchenko, M., Hanasz, J., and Rucker, H. O.: Estimation of linear wave polarization of the auroral kilometric radiation, Radio Sci., 43, RS1006, doi:10.1029/2006RS003606, 2008.

Uozumi, T., Yumoto, K., Tokunaga, T., Solovyev, S. I., Shevtsov, B. M., Marshall, R., Liou, K., Ohtani, S., Abe, S., Ikeda, A., Kitamura, K., Yoshikawa, A., Kawano, H., and Itonaga, M.: AKR modulation and global Pi2 oscilation, J. Geophys. Res., 116, A06214, doi:10.1029/2010JA016042, 2011.

$\mathrm{Wu}, \mathrm{C}$. S. and Lee, L. C.: Theory of the terrestrial kilometric radiation, ApJ., 230, 621-626, doi:10.1086/157120, 1979.

Zarka, P.: Auroral radio emissions at the outer planets: observations and theories, J. Geophys. Res., 103, 20159-20194, 1998. 